Cita bibliográfica: Peña Sánchez, A. R., Jiménez García, M., Ruiz Chico, J. y Pontón Aricha, T. (2019). La producción científica en el "Sector de Hostelería y Turismo": Un análisis bibliométrico de las tesis doctorales españolas en el periodo 1978-2018. Investigaciones Turísticas (18), pp. 71-94. http://dx.doi.org/10.14198/INTURI2019.18.04

\title{
La producción científica en el "Sector de Hostelería y Turismo": Un análisis bibliométrico de las tesis doctorales españolas en el periodo 1978-2018
}

\section{Scientific production on "Hospitality and Tourism Sector": An analysis of the spanish doctoral theses in the period 1978-2018}

\author{
Antonio Rafael Peña Sánchez (iD, Universidad de Cádiz, España \\ rafael.pena@gm.uca.es
}

Mercedes Jiménez García, Universidad de Cádiz, España mercedes.jimenezgarcia@uca.es

José Ruiz Chico, Universidad de Cádiz, España jose.ruizchico@uca.es

Teresa Pontón Aricha, Universidad Pablo de Olavide, España tponari@upo.es

\section{RESUMEN}

El objetivo principal de este trabajo es desarrollar un estudio descriptivo, detallado y pormenorizado de la producción científica catalogada bajo el descriptor denominado "Sector Hostelería y Turismo" recogidas en las tesis doctorales inscritas en la base de datos TESEO, con la finalidad de cuantificar y trazar de forma cronológica la producción de tesis doctorales por universidades, directores y participación en tribunales, sexo, e idioma. Se trata de identificar algunos de los principales actores que han protagonizado la investigación científica, ofreciendo una panorámica pormenorizada del progreso que se ha ido produciendo en los trabajos catalogados bajo este descriptor, lo que sin duda favorecerá la comprensión de la evolución en este campo del conocimiento científico en los últimos años.

Palabras claves: hostelería, turismo, tesis doctorales, producción científica, investigación científica.

\section{ABSTRACT}

The main objective of this research is to develop a descriptive and detailed study of the scientific production classified according to the descriptor "Hospitality and Tourism Sector" included in the doctoral theses registered in the TESEO database, in order to quantify and chronologically trace the production of doctoral theses by universities, directors and participation in 
committees, gender, and language. The aim is to identify some of the main agents that have carried out scientific research, offering a detailed overview of the progress that has taken place in the studies catalogued under this descriptor, which will undoubtedly favour the understanding of the evolution in this field of scientific knowledge in recent years.

Keywords: hospitality, tourism, doctoral theses, scientific production, scientific research.

\section{INTRODUCCIÓN}

Recientemente se viene experimentando un incremento del interés que suscita el turismo como área de investigación de la Ciencia Social, reflejado en la proliferación del número de trabajos publicados tanto a nivel nacional, como a nivel internacional. Este fenómeno ha tenido su paralelismo con la evolución experimentada por la situación económica de este sector. En efecto, la industria del turismo actualmente constituye un activo motor del desarrollo económico en muchos países, sobre todo en los desarrollados y emergentes (Nel-lo y Pérez, 2007; Moreno y Picazo, 2012). A nivel mundial, según el informe de la World Travel \& Tourism Council (WTTC) (Hostelsur, 2018), la producción directa de la industria turística fue de 2,4 billones de dólares en el año 2014, lo que representó el 3,1 \% del Producto Interior Bruto (PIB) mundial, y generó 105 millones de empleos. No obstante, si se suman los efectos de arrastre o indirectos, el montante generado fue de 7,6 billones de dólares, lo que suponía el 9,8 \% del PIB mundial. Tal es su importancia, que el volumen de negocios de esta actividad económica tuvo un impacto superior al de la industria química $(8,6 \%)$, la agricultura $(8,5 \%)$, la educación $(8,4 \%)$, la industria automovilística $(7,0 \%)$ y la actividad bancaria $(5,9 \%)$ a nivel mundial. En la misma línea, según la Organización Mundial del Turismo (OMT, 2017), un número cada vez mayor de destinos de todo el mundo se han abierto recientemente al turismo, haciendo del mismo un sector muy dinámico y esencial para el progreso socioeconómico. De hecho, se ha observado un crecimiento prácticamente ininterrumpido, a pesar de las crisis económicas y financieras sufridas, mostrando regularmente su fortaleza y resistencia, tal y como se señala en la literatura económica al respecto (Torres, 1994; Massieu, 2004; Esteban, 2004; Rosselló et al., 2005; Flores y Barroso, 2012; Jiménez et al., 2015a, 2016).

Europa sigue siendo la primera región turística a nivel mundial en 2016, tanto por llegadas como por ingresos de turismo internacional. Y los países europeos más destacados turísticamente son Francia y España, que ocupan la primera y la segunda posición en el ranking de los principales destinos turísticos por llegadas de turistas internacionales (con 82,6 y 75,5 millones de visitantes, respectivamente). Sin embargo, la situación se invierte en cuanto a ingresos por turismo internacional, siendo España la que mayores ingresos genera (60 mil millones de dólares, frente a los 42,5 mil millones de Francia).

El turismo, por tanto, se configura como un sector estratégico en la economía española a nivel mundial. En este sentido, la producción del sector fue de 125.529 millones de euros en 2016 , un $6,6 \%$ más que en 2015 , volumen que supone el $11,2 \%$ del PIB español, según la Cuenta Satélite del Turismo de España (2010-2016), publicada por el Instituto Nacional de Estadística (INE). Además, generó 2,56 millones de empleos en el mismo año, cifra equivalente al $13 \%$ del empleo total, mientras que el mismo porcentaje fue de 12,7 \% en 2015. 
Lo anterior podría haber provocado, sin duda, que los académicos e investigadores de esta rama del conocimiento hayan marcado un rumbo creciente en el número de trabajos publicados en los últimos años. Así, este fenómeno puede haber favorecido el auge del conocimiento y los análisis en esta área de conocimiento en la investigación científica española, tal y como se tratará de poner de manifiesto a lo largo de este documento.

En este sentido, este trabajo pretende realizar un estudio bibliométrico descriptivo y riguroso de la producción científica catalogada bajo el descriptor denominado "Sector Hostelería y Turismo" recogidas en las tesis doctorales inscritas en la base de datos TESEO, mediante un examen detallado, con el objetivo de cuantificar y trazar de forma cronológica la producción de tesis doctorales por universidades, directores y participación en tribunales, sexo, e idioma, identificando algunos de los principales actores que han protagonizado la investigación científica, ofreciendo una panorámica pormenorizada de los cambios, así como del progreso que se ha ido produciendo en los trabajos catalogados bajo este descriptor, lo que sin duda favorecerá la comprensión de la evolución experimentada en los últimos años.

La enorme utilidad de la bibliometría permite reflexionar sobre los estudios realizados en un campo del conocimiento concreto, con el objeto de conocer las personas e instituciones relacionadas con un área concreta de investigación, y con el fin de poder evaluar el desempeño de dichas personas e instituciones (Hall, 2011). El interés de este trabajo se centra, pues, en la información que se desprende de la evaluación de la investigación sobre el sector turístico y hostelero en España, pues aporta relevantes consideraciones para diferentes colectivos como pueden ser los profesionales del sector, académicos, investigadores, futuros doctores e instituciones interesadas en el área, arrojando reflexiones actualizadas de carácter científico sobre la producción científica en este campo del saber.

Este trabajo se inicia con una revisión de la literatura existente sobre la cuestión objeto de estudio. A continuación, se realiza una breve descripción de la metodología empleada y la justificación de la fuente estadística utilizada. En el cuarto apartado se exponen de forma detallada los resultados obtenidos en el análisis llevado a cabo. Finalmente, se presenta un apartado con la discusión del análisis realizado y las conclusiones obtenidas.

\section{REVISIÓN DE LA LITERATURA}

La bibliometría constituye la parte del conocimiento científico que se encarga de la medida, a través de indicadores, del número de documentos publicados y de las citas que estos trabajos reciben, en función de su origen geográfico y su conjunto de autores, lo que permite la evaluación del producto de la Ciencia (Cortés, 2007). El análisis bibliométrico se apoya en la utilización de métodos cuantitativos y el tratamiento de indicadores como el número de científicos y la cuantía y evolución de los trabajos científicos, mostrándose recientemente como una de las ramas más prolíficas de la investigación documental (Salvador, 2007). Es una herramienta esencial para describir y evaluar los resultados de la producción científica y la productividad en distintas áreas del conocimiento. En este sentido, Das y Handfield (1997) señalan que las tesis doctorales representan una vía práctica para valorar las aportaciones conceptuales y empíricas llevadas a cabo en un campo científico concreto. En efecto, las tesis doctorales cubren determinadas lagunas no tratadas o indicadas en otros documentos de investigación, 
dando lugar en ocasiones a posteriores publicaciones en revistas científicas (Jeong, 2001). Así, Ortega y Rodríguez (2004) sostienen que las tesis doctorales constituyen una importante aportación investigadora en los campos en los que se realizan, y concretamente en la actividad turística, reflejándose en la producción creciente que ha experimentado ésta a lo largo de los años (Hernández et al., 2011). En este mismo sentido se pronuncian Esteban (2000), Ortega et al. (2003), Ortega y Rodríguez (2004), López y López (2008) y Vargas (2011a), cuando señalan la evolución experimentada por la investigación científica del turismo en los últimos tiempos. Y no es aventurado afirmar que el mayor interés relativo del turismo en la investigación de las tesis doctorales en España se encuentra correlacionada con la importancia de la actividad turística en la actividad económica española (Agencia Valenciana del Turismo, 2006). En efecto, la formación y la investigación en el campo turístico han progresado paralelamente al crecimiento del sector (Vera e Ivars, 2001), lo que ha permitido que el trabajo científico en el sector hostelero y turístico haya evolucionado con enorme virulencia en las últimas décadas (Precedo et al., 2007; Navarro y Vejsberg, 2009; Helmsing y Ellinger, 2011; Jiménez et al., 2015b, 2017). Aunque algunos autores se cuestionan si es comparable la importancia que se le da a la investigación en este campo del conocimiento en España, Esteban (2000) sostiene que la histórica escasa consideración social, política y académica del turismo ha impedido la equiparación de un cuerpo de investigación científica en esta materia con su importancia económica y empresarial. Del mismo modo, Vargas (2011a) afirma que el estudio de este sector cuenta con una escasa tradición académica, y enfatiza que es precisamente después de la aparición de la formación universitaria del turismo en 1996, con el Real Decreto 259/1996, de febrero, que regula la incorporación de las enseñanzas turísticas a la Universidad, cuando comienza a proliferar los trabajos científicos en esta área del conocimiento, tratando básicamente aspectos económicos y geográficos. En efecto, las investigaciones universitarias del aspecto turístico reflejan consecuentemente la evolución de esta actividad en el mundo en general (López-Bonilla et al., 2017), y en la sociedad española en particular. No obstante, a pesar de la consolidación de los estudios turísticos a nivel nacional e internacional (Albacete y Fuentes, 2010; Albacete, Fuentes y Haro-Domínguez, 2013), muchas de las publicaciones siguen considerando esta actividad, más que como una disciplina propia, como un campo de estudios asociado a otras disciplinas, como el la Organización y Dirección de Empresas, Marketing, Recursos Humanos, Ordenación del Territorio, Geografía, etc. (Moreno y Picazo, 2012; Durán et al., 2015).

Con estos antecedentes, los cambios que en los últimos años está experimentando el sector turístico y la hostelería hace que la Universidad necesite adaptarse a los mismos, aumentando la calidad de las investigaciones que se realizan, así como la visibilidad y transferencia de los conocimientos que se desprenden de dichos trabajos científicos (Bermúdez et al., 2009; Conferencia de Rectores de Universidades Españolas, 2013: 244). Este hecho ha repercutido en que en los últimos años se hayan publicado numerosos trabajos científicos en el campo del turismo y la hostelería, entre los cuales se encuentran las tesis doctorales, consideradas como uno de los principales indicadores de la investigación universitaria, y constituyendo el primer encuentro científico entre los investigadores y las materias de estudio en el ámbito académico (Esteban, 2000). Las tesis doctorales se definen como un trabajo de investigación inédito referido a una materia científica, técnica o artística, que se realiza una vez concluido un periodo docente orientado a la preparación del investigador (Colás et al., 
2009). Ello supone la culminación del ciclo de aprendizaje e investigación de un científico en formación bajo la dirección o tutela de uno o varios directores de tesis. Al ser trabajos originales y muy especializados, que aportan nuevos conocimientos en el área investigada, cabe reivindicar su importancia científica pues proporcionan una imagen fiel de las nuevas vías de investigación (Muñoz, 2005), además de la completa revisión bibliográfica que suele contener (Miguel, 2000), lo que permite un primer contacto del doctorando con las materias susceptibles de investigación. Consecuentemente, dada la importancia académica de estos trabajos científicos, los estudios de doctorado en España han estado regulados por distintos Reales Decretos (RD), siendo los más recientes el RD 778/1198, sustituido por el RD 56/2005 y luego el RD 1393/2007, en el que se incorporan aspectos referentes al Doctorado Europeo o con Mención de Calidad (Ortiz-Sánchez y Martín-Moreno, 2011). Además, la aparición y consolidación de grupos de investigación dedicados a este ámbito de estudio y asociaciones como AECIT (Asociación Española de Expertos Científicos en Turismo), promovidas desde un entorno académico, ha posibilitado la puesta en común de los resultados de investigación obtenidos, contribuyendo a un avance más colaborativo del conocimiento del sector turístico (CorralMarfil y Cánoves-Valiente, 2016). Por ende, simultáneamente al crecimiento de la actividad turística, la investigación ha ido alcanzando un tamaño relevante y un reconocimiento explícito en las últimas décadas, como campo del conocimiento multidisciplinar inmerso en las Ciencias Sociales (Zhao y Richie, 2007; Xiao y Smith, 2008; Racherla y Hu, 2010).

\section{METODOLOGÍA Y FUENTES ESTADÍSTICAS}

Este trabajo de investigación trata de caracterizar la producción científica española de tesis doctorales que se han adscrito al descriptor "Sector de Hostelería y Turismo" del Tesauro, con el código 531213 de normalización UNESCO. Se ha seleccionado este sistema de codificación por tratarse de un criterio consistente en una nomenclatura internacional para las áreas de ciencia y tecnología, utilizada desde 1973 en España, y por el Ministerio de Ciencia y Tecnología desde 1983 en virtud de la Resolución de 23 de septiembre del mismo. El número de trabajos registrados fue uno de los elementos determinantes al hacer el estudio de este único descriptor, lo que deja una línea de investigación abierta a futuros trabajos que deseen cruzar con otros descriptores del mismo campo. En efecto, resulta interesante tener en cuenta la limitación que supone tomar una determinada fuente de información al concretar un perfil de búsqueda. Los resultados de este trabajo se deben contextualizar dentro de la base estadística seleccionada, es decir, Teseo, en el presente caso, que recoge la producción científica de trabajos con un alto nivel de profundización investigadora en los aspectos analizados. Actualmente Teseo es la principal fuente de información de las tesis aprobadas en las universidades españolas. Desde su aparición en 1976, esta base de datos posibilita la búsqueda de trabajos científicos por múltiples criterios, como el título, el autor, el director, la universidad, el curso académico, el departamento, el resumen y las palabras claves, en la que la última versión está habilitada para ser utilizada como un sistema de gestión de tesis doctorales a nivel nacional (Fuentes y Arguimbau, 2010).

La metodología empleada, siguiendo a Moreno-Fernández y Moreno-Crespo (2016), se basa en 4 fases de investigación, a saber: a) documentación, b) obtención, tratamiento, organización y tabulación de los datos, c) análisis de la información, y d) elaboración y difusión 
del informe final con los resultados obtenidos. Para la recuperación de la información se ha acudido a la base de datos de TESEO, dependiente del Ministerio de Educación, Cultura y Deporte, cuya finalidad es recopilar los registros de las tesis defendidas en las universidades españolas. La selección de esta base de datos está justificada por su amplio recorrido cronológico y la detallada descripción que presentan sus registros bibliográficos, incluyendo datos de enorme interés como es la composición de los tribunales, siendo este factor una fuente recurrente en los análisis españoles sobre las tesis doctorales (Fernández-Cano et al., 2008). No obstante, algunos autores han puesto de manifiesto las limitaciones de dicha fuente de datos, sobre todo con aspectos relacionados con la normalización de sus registros (Fuentes y Arguinbau, 2010; Repiso et al., 2011; Martínez, 2011a y 2011b). En relación a su cobertura, es necesario decir que no recoge el total de tesis existentes, pues las que no fueron remitidas por la universidad o por el doctorando no figuran en el catálogo. Pero a pesar de ello, lo cierto es que es la única herramienta que recoge con mayor rigurosidad el conjunto de tesis doctorales de las universidades españolas, además de ser prácticamente la única que se utiliza en los estudios bibliométricos de corte cuantitativo (Soli y Merlo, 2002). Es por ello por lo que en este trabajo se ha decidido por esta base de datos. A pesar de lo anterior, esta investigación puede ser un avance que permita suscitar un interés en futuras líneas de investigación que analicen otros trabajos documentales como artículos, comunicaciones en congresos y reuniones especializadas, informes, ect., publicados con otras bases de datos, lo que sin duda posibilitaría la ampliación y profundización de la presente investigación.

Los registros obtenidos en Teseo bajo la denominación de "Sector de Hostelería y Turismo" presentan 527 trabajos de investigación dentro del criterio codificado por el identificador 531213, convirtiéndose ésta en la muestra con la que se ha realizado el estudio. El periodo analizado comprende desde 1978 a 2018 (concretamente el último registro se obtuvo en marzo de 2018, periodo en el que se cerró la muestra para el análisis bibliométrico), por ser precisamente el espacio cronológico en el que se incluye la totalidad de las tesis doctorales incluidas en el identificador considerado en la fuente de información utilizada. Los datos se extrajeron y recopilaron un inventario de referencias creado para su tratamiento, cuantificación y obtención de resultados. Los aspectos comúnmente analizados en este tipo de trabajos suelen ser los indicadores temáticos (disciplinas y áreas de estudio, que en el caso concreto de este estudio está perfectamente delimitado) y los de productividad (directores de tesis, tribunales, universidades y centros de lecturas y años). Además, otras variables que se suelen tratar en este tipo de investigaciones son el sexo del doctorando, del director/a y codirector/a de tesis, de los miembros del tribunal, y el idioma de lectura. Los registros creados para el presente trabajo fueron: nombre y apellidos del autor/a, sexo, título de la tesis doctoral, año académico en el que fue leída, universidad en la que se defendió, nombre y apellidos del director/a o directores/as de la tesis, los miembros que formaron el tribunal que juzgó a la tesis, idioma de presentación y posesión o no de la mención europea o internacional del título.

\section{RESULTADOS OBTENIDOS}

\section{Evolución de la producción científica}

Las primeras tesis doctorales en el campo científico del turismo datan de finales de los años cincuenta, leídas en la denominada Universidad de Madrid en aquella época, y actual 
Universidad Complutense de Madrid. En esa época se presentaron dos tesis doctorales tituladas "La industria turística en la economía nacional y sus expectativas", en 1958, y otra cuyo título fue "El turismo social en España", defendida un año después (Ortega y Rodríguez, 2004). Estos trabajos seminales marcaron el inicio de un ámbito de investigación que posteriormente ha ido ampliándose y extendiéndose, con la adscripción del Turismo a las Ciencias Sociales. Estas tesis doctorales no aparecen en la base de datos Teseo porque, como se ha indicado anteriormente, la incorporación de registros no comenzó hasta 1976.

El número de tesis doctorales defendidas en el periodo 1978-2018 registradas en la base de datos TESEO para el descriptor "Sector Hostelería y Turismo" han sido de 527, que son las que han sido examinadas en el presente trabajo. Desde que en 1978 se expusieran por sus autores las primeras tesis registradas bajo esta denominación, a saber, la tesis de D. Francisco Jurado Arrones titulada "Turismo residencial y colonización" y la de D. Manuel Figuerola Palomo con el título "Elementos de una teoría económica del turismo y métodos para su análisis cuantitativo", la progresión temporal presentada por las tesis registradas en la base de datos del Ministerio de Educación, Cultura y Deporte del Gobierno de España ha sido muy peculiar, además de irregular cronológicamente, ya que no es hasta el periodo 1998-2003 en el que se defienden un total de 83 tesis científicas y empieza a surgir una plétora de trabajos inéditos sobre este campo científico. No obstante, es a partir de 2008 cuando se consolida el proceso expansivo de la producción científica del sector objeto de estudio, tal y como se puede observar en la tabla 1 y la figura 1, llegando a defenderse 56 tesis en 2014, 57 en 2015, 93 en 2016 y 54 en 2017, lo que supone un total de 260 tesis doctorales, prácticamente la mitad de las tesis doctorales leídas a lo largo del dilatado periodo analizado 1978-2018. Estos resultados concuerdan con los obtenidos por otros investigadores del análisis turístico en el campo de la economía y la dirección de empresas (Albacete y Fuentes, 2010). Y, según Ceballos et al. (2010), los motivos señalados para el crecimiento de la investigación turística han podido ser el continuo crecimiento de la actividad turística en España y en el mundo, el aumento del interés internacional por el estudio de este aspecto social y la creación y modificación de los estudios universitarios de grado, máster y doctorado en las universidades.

Tabla 1

Número de tesis por periodos de tiempo y por sexos

\begin{tabular}{|c|c|c|c|}
\hline Periodo & No Tesis & Mujeres & Hombres \\
\hline $1978-1988$ & 6 & 1 & 5 \\
\hline $1989-1998$ & 15 & 5 & 10 \\
\hline $1999-2008$ & 95 & 46 & 49 \\
\hline $2009-2018$ & 411 & 211 & 200 \\
\hline TOTAL & $\mathbf{5 2 7}$ & $\mathbf{2 6 3}$ & $\mathbf{2 6 4}$ \\
\hline
\end{tabular}

FUENTE: Elaboración propia a partir de los datos de la Base de datos TESEO.

Si se divide el dilatado periodo analizado de 40 años en espacios temporales de 10 años, se observa claramente como es a partir de 1999 cuando comienzan a intensificarse la productividad científica en esta rama del conocimiento, siendo a partir de 2009 el periodo en el 
que se produce una verdadera eclosión en el número de tesis doctorales leídas, lo que supone el $78 \%$ de las tesis doctorales defendidas en el espacio temporal analizado. Con respecto a las tesis leídas por mujeres y hombres, es también a partir del año 2009 cuando se produce una fuerte eclosión en la participación de la mujer en la investigación científica a través de las tesis doctorales en esta rama del conocimiento. La primera tesis doctoral defendida por una mujer dentro de este campo científico data de 1988, siendo la autora Dña. Alfonsa Denia Cuesta con la tesis titulada "Análisis estructural y predictivo de la demanda turística en España". Desde este momento, no es hasta diez años después, es decir 1998, cuando comienza una producción regular de tesis doctorales por parte del género femenino. A pesar de la tardía aparición de tesis doctorales en el "Sector de Hostelería y Turismo" por parte de las mujeres, resulta interesante poner de manifiesto como hasta el momento, de las 527 tesis doctorales registradas en la base de datos analizada, 263 corresponden a mujeres, y 264 a hombres, lo que supone una producción científica de la mitad de tesis doctorales en las tres cuartas partes del periodo analizado, lo que indudablemente refleja la intensa productividad experimentada por la mujer en este campo científico. Además, cabe resaltar como en la última década el número de registros que presenta la base de datos Teseo es superior en el caso de la mujer (211 tesis leídas) frente al caso de hombres (200 tesis leídas), así como que en la década anterior la situación era muy equitativa, con 46 tesis doctorales de mujeres y 49 tesis de hombres.

Con respecto a las disciplinas de investigación en las que se han desarrollado estos trabajos, las más representativas han sido la Economía y la Empresa (en sus distintas vertientes, como la organización de empresas, los recursos humanos y el marketing) y la Geografía.

Figura 1

Evolución del número de tesis doctorales defendidas y por sexos (1978-2018*)

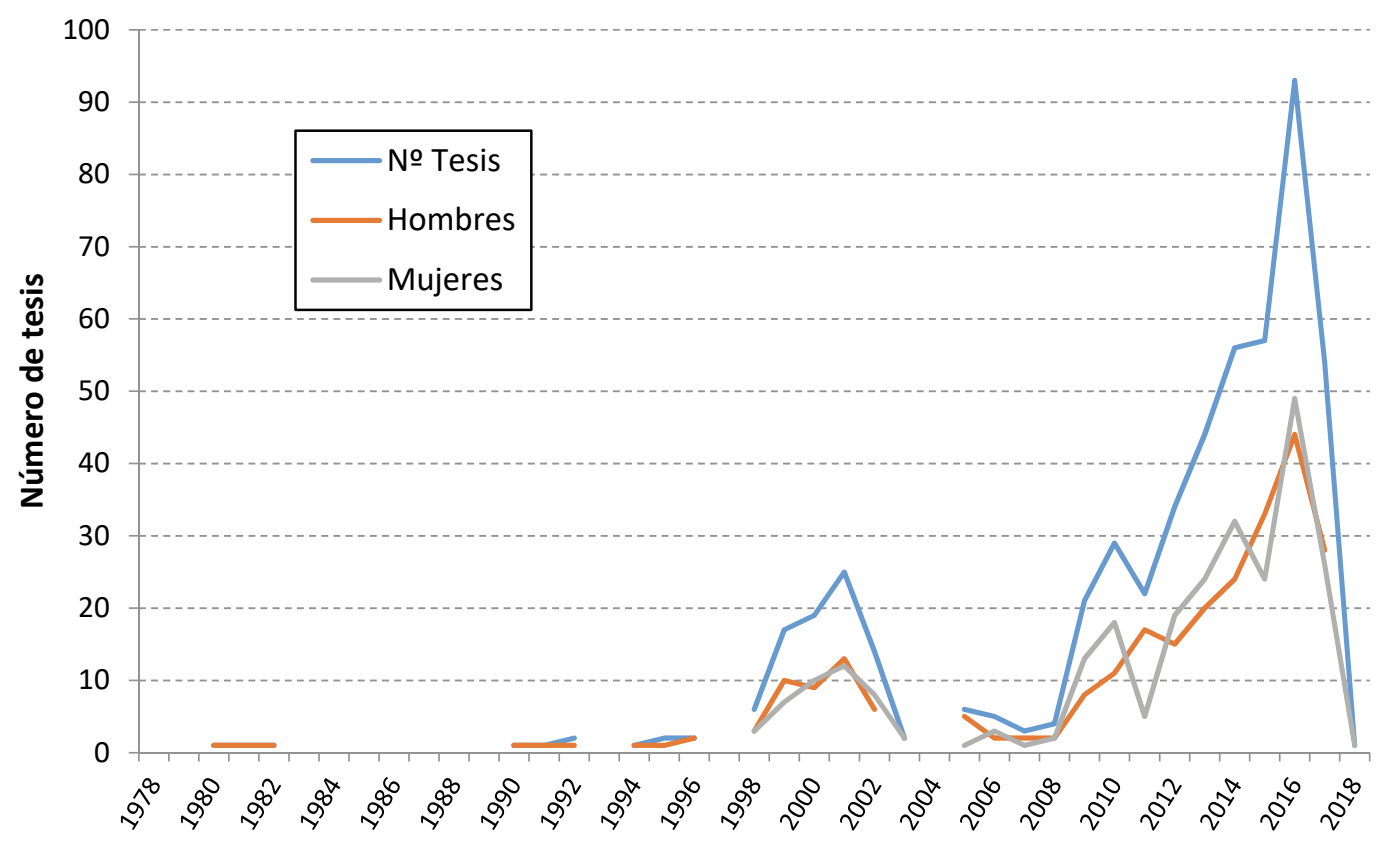

NOTA: (*) Último dato extraído de la base de datos el 20 de marzo de 2018.

FUENTE: Elaboración propia a partir de los datos de la Base de datos TESEO. 
Las universidades en las que se han defendido dichos trabajos inéditos de investigación son 61 , de las cuales hay algunas con una enorme productividad en esta área de conocimiento, como pueden ser la Universidad de Málaga (con 62 tesis doctorales registradas), Universidad de las Illes Balears (con 48), Universidad de Alicante (con 27), Universidad de La Laguna (con 26), Universidad de Girona (con 25), y Universidad de Sevilla (con 20), lo que supone un total de 208 tesis doctorales, es decir, casi un $40 \%$ de las tesis defendidas en las universidades españolas en el periodo 1978-2018 (véase Anexo I). Por tanto, se puede afirmar como existe una elevada concentración de tesis en pocas universidades españolas, de tal manera que tan sólo cuatro universidades españolas congregan más del $30 \%$ de las tesis doctorales defendidas en el periodo analizado, mientras que 28 universidades, las menos productivas en esta línea científica, sólo producen alrededor del $10 \%$ de las tesis doctorales en este campo del conocimiento. En el mismo sentido, el número medio de tesis doctorales sobre turismo defendidas al año por las 5 universidades más productivas en este campo del conocimiento fue de 4,7 tesis, mientras que la media de las 12 universidades menos productivas fue de 0,3 tesis al año, observándose como las universidades con mayor productividad en la investigación turística se encuentran ubicadas en zonas geográficas con una participación económica significativa del sector turístico en su actividad económica.

Si se centra la atención en aquellas universidades en las que se han leído más de 15 tesis doctorales en el periodo analizado, se debe señalar también a la Universitat Politécnica de Valencia (con 19 tesis defendidas), Universidad de Extremadura (con 18), y Universidad de Antonio de Nebrija y Universidad de Santiago de Compostela (con 16 tesis cada una). En total, en las universidades más productivas en este campo científico, es decir, aquellas que acumulan más de 15 tesis doctorales en el periodo analizado, se han evaluado un total de 277 tesis, lo que supone cerca del $53 \%$ de las tesis doctorales totales.

El castellano es el idioma generalmente empleado por la inmensa mayoría de los autores de tesis defendidas en el periodo analizado, pues de las 527 tesis registradas según la base de datos examinadas 474 han sido presentadas y leídas frente al tribunal en español, lo que supone cerca de un $90 \%$ de las tesis totales registradas.

Del total de tesis calificadas en las universidades españolas según la base de datos Teseo, $\mathbf{4 8}$ han sido presentadas en una lengua distinta al castellano. La primera tesis presentada en idioma no castellano fue la de Dña. Ana María Munar Chacartegui, titulada "Tourism education \& globalization. The Bologna Process" en el año 2006, justo después de la puesta en vigor del Real Decreto 56/2005, de 21 de enero, por el que se regulan los estudios universitarios oficiales de Posgrado. Además, de todas las tesis defendidas en el periodo en cuestión 44 han obtenido la Mención Europea o Mención Internacional, tal y como regulan el RD 1393/2007, de 29 de octubre, por el que se establece la ordenación de las enseñanzas universitarias oficiales y el RD 99/2011, de 28 de enero, por el que se regulan las enseñanzas oficiales de doctorado, de las que 24 fueron defendidas por hombres y 20 por mujeres. 
Tabla 2

Número de tesis por lenguas, sexos y mención

\begin{tabular}{|l|c|c|c|c|}
\hline Lengua & No Tesis & $\begin{array}{c}\text { Autoría } \\
\text { Hombre }\end{array}$ & $\begin{array}{c}\text { Autoría } \\
\text { Mujer }\end{array}$ & $\begin{array}{c}\text { Mención } \\
\text { Europea o } \\
\text { Internacional }\end{array}$ \\
\hline Español & 474 & 240 & 234 & 36 \\
\hline Inglés & 30 & 16 & 14 & 5 \\
\hline Catalán & 9 & 4 & 5 & 0 \\
\hline Portugués & 6 & 2 & 4 & 2 \\
\hline Español/Inglés & 4 & 1 & 3 & 1 \\
\hline Gallego & 2 & 1 & 1 & 0 \\
\hline Español/Gallego & 1 & 0 & 1 & 0 \\
\hline Francés & 1 & 0 & 1 & 0 \\
\hline TOTAL & $\mathbf{5 2 7}$ & $\mathbf{2 6 4}$ & $\mathbf{2 6 3}$ & $\mathbf{4 4}$ \\
\hline
\end{tabular}

FUENTE: Elaboración propia a partir de los datos de la Base de datos TESEO.

Con respecto a las tesis presentadas en idiomas distintos, se puede observar como en inglés y portugués se han leído 36 tesis, de las cuales 18 han sido defendidas por mujeres y 18 por hombres. En Español/Inglés han sido presentadas 4, repartidas al $25 \%$ entre hombres y $75 \%$ en mujeres. En francés fue presentada una tesis, y lo hizo una mujer, concretamente en el año 2016.

\section{Direcciones de tesis}

Un aspecto interesante del estudio bibliométrico es poder poner de manifiesto quien ha sido el profesorado que más tesis doctorales han dirigido en este campo científico. En este sentido, en el Anexo II se presentan todos aquellos directores que, a lo largo del periodo analizado, se registran en la base de datos de TESEO como los directores de tesis que han dirigido a dos o más doctorandos. Concretamente, si consideramos aquellos/as directores/as con más de tres tesis dirigidas, la mayor productividad en la dirección de tesis en el periodo analizado en esta rama del conocimiento viene constituida por 100 tesis doctorales, lo que representa casi el $19 \%$ del total de las tesis analizadas, dirigidas por 21 profesores universitarios. Sin embargo, en el caso de incluir a los/as directores/as con más de 2 tesis dirigidas, el número de duplica, llegando a 203 tesis, lo que supone el 38,5 \% del total de tesis que conforma la población estudiada, incrementándose en 50 más el número de directores/as de tesis con mayor productividad.

El listado de Profesores con mayor volumen de direcciones de tesis viene encabezado por el Profesor Torres Bernier, que ha dirigido 13 tesis doctorales en la Universidad de Málaga. Es destacable que en la misma Universidad, la Profesora Tous Zamora también llevó a cabo la labor de dirección en 4 tesis doctorales. Al Profesor Torres Bernier le sigue el Profesor Vera Rebollo, con 9 tesis doctorales dirigidas en el Sector Hostelería y Turismo, en la Universidad de Alicante, y el Profesor Guía Julve, con 8 tesis doctorales, de las cuales 7 fueron en la Universidad de Girona y una en la Universitat Oberta de Catalunya. 
De las 203 tesis consideradas en este apartado, como tesis dirigidas por el profesorado que cuentan con más tesis dirigidas, 13 han obtenido la Mención Europea o Internacional.

Con respecto a la dirección y codirección de las tesis, se observa como de las 527 tesis analizadas, 301 tenían un único director/a de tesis, y 226 han sido codirigidas. En este sentido, resulta interesante poner de manifiesto como no es hasta 1998 cuando aparecen dos tesis codirigidas. Anteriormente sólo aparecen tesis con un/a sólo/a director/a. Pero no es hasta el año 2009 en el que se comienza a vislumbrar una tendencia creciente en la codirección de las tesis doctorales de este campo de conocimiento, llegando a aparecer algunos años en el que el número de tesis con codirector/a era superior al de tesis con un/a único/a director/a, como el 2012 (22 tesis codirigidas frente a 12 tesis dirigidas), 2014 (35 tesis codirigidas frente a 21 tesis dirigidas) y 2017 (28 tesis codirigidas frente a 26 tesis dirigidas). Ello podría estar indicando que en los análisis en este campo del conocimiento se viene creando una colaboración entre el profesorado doctor a la hora de dirigir este tipo de trabajos, lo cual podría ser interesante ya que las ideas que aportan los codirectores en la codirección de estas tesis favorece el trabajo investigador llevado a cabo por el doctorando.

Por tanto, se observa que en este campo científico existe un elevado número de profesores y profesoras que han desarrollado la dirección de más de dos tesis doctorales, lo que en cierta manera muestra no sólo el interés en esta rama del conocimiento sino además en el beneficio que proporciona la evolución del desarrollo científico en esta área de investigación.

Con respecto al sexo de los/as directores/as de tesis, resulta importante destacar como en el periodo analizado, de las 527 tesis consideradas en el estudio, 365 fueron dirigidas por hombres, lo que supone el $69 \%$ del total, y 139 fueron dirigidas por mujeres, es decir, el $26 \%$. La primera directora de tesis en este campo, según la base de datos utilizada, fue la Profesora Doctora Dña. Amparo Sancho Pérez, cuya lectura se llevó a cabo en el año 1996. Y a partir de este año, la proliferación de directoras de tesis fue considerablemente importante.

Las codirecciones de tesis comienzan en este campo del saber en el año 1998, y a partir de este año el número de tesis codirigidas comienza a ser más relevante. El número de tesis codirigidas por hombres fue de 131, mientras que las codirigidas por mujeres fue de 73, es decir, prácticamente la mitad. Este dato resume en cierta manera la falta de protagonismo que hasta el momento se le ha dado a la mujer en las direcciones y codirecciones de tesis. No obstante, es cierto que dicho protagonismo va siendo cada vez mayor en los últimos años del periodo analizado, disminuyendo el desequilibrio de la participación entre hombres y mujeres en este caso.

\section{Presidencias de Tribunal}

En este apartado se presentan los presidentes y presidentas de tribunales de tesis que han pertenecido a más de 3 tribunales (véase Anexo III). La totalidad de tribunales presididos fueron de 143, lo que supone el $27 \%$ de la totalidad de tesis defendidas sobre el sector en cuestión en el periodo analizado.

El Profesor Aguiló Pérez fue el que, con diferencia, más veces ocupó el puesto de presidente en los tribunales que han juzgado las tesis doctorales correspondiente a este campo 
científico. Presidió 18 tribunales, en diferentes Universidades, como la Universidad de Jaén (3 tribunales de tesis doctorales), la Universidad de las Illes Balears (11), la Universidad de Málaga (2), la Universidad de Oviedo (1) y la Universidad Jaume I de Castellón (1). Le siguen, a una distancia relevante, el Profesor Vera Rebollo, que ha presidido 9 tribunales de tesis, pero muy repartidas entre las universidades españolas. De facto, presidió en la Universidad Antonio de Nebrija (1 tribunal de tesis doctoral), en la Universidad de Alicante (1), en la Universidad de Girona (1), en la Universidad de Málaga (2), en la Universidad de Oviedo (1), en la Universidad de Sevilla (1), en la Universidad de Vigo (1) y en la Universitat Politécnica de Valencia (1). Y le sigue la Profesora Parra Guerrero, que presidió 7 tribunales de tesis, todas ellas en la Universidad de Málaga.

Por tanto, se observa como existe un profesorado muy implicado en este campo científico, que ha invertido gran parte de su tiempo investigador en la lectura minuciosa y posterior evaluación y valoración de tesis doctorales sobre Hostelería y Turismo.

Si tenemos en cuenta las cuestiones de género en este apartado, resulta relevante poner de manifiesto como de las tesis consideradas en el estudio, en 432 estuvo como presidente de tribunal un hombre, y tan sólo en 94 estuvo como presidenta una mujer. De hecho, la primera presidenta de tribunal en una tesis de este campo del conocimiento fue la Profesora Doctora Dña. Alegría Borrás Rodríguez, en el año 1995. Con respecto a los vocales, se puede decir que la participación de hombres fue mayor que el de mujeres en el periodo estudiado. Y en el caso de la Secretaría del Tribunal, 329 casos estuvieron participadas por un hombre y 196 por una mujer. Por lo que, de nuevo, se pone de manifiesto las enormes disparidades de género que han existido en los miembros que han conformado los tribunales de tesis en esta área de investigación. No obstante, resulta relevante destacar como la participación de la mujer ha ido incrementándose de forma sustancial en los últimos años del periodo analizado, reduciendo significativamente las disparidades existentes en la contribución de la mujer en los tribunales de tesis.

\section{DISCUSIÓN Y CONCLUSIONES}

Este trabajo ha tratado de realizar un análisis de la producción científica catalogada bajo el descriptor denominado "Sector Hostelería y Turismo" recogidas en las tesis doctorales inscritas en la base de datos TESEO en el periodo 1978-2018, utilizando para ello métodos bibliométricos, con el objetivo principal de cuantificar y trazar de forma cronológica la producción de tesis doctorales por universidades, directores y participación en tribunales, sexo, e idioma, identificando algunos de los principales actores que han protagonizado la investigación científica, ofreciendo una panorámica pormenorizada de los cambios, así como del progreso que se ha ido produciendo en los trabajos catalogados bajo este descriptor, y reflejando la incidencia de los cambios normativos experimentados en la legislación del doctorado cuando ha sido necesario.

Así, en las últimas décadas, y a pesar de los inconvenientes por los que ha pasado la economía española en general y la economía del sector hostelería y turismo en particular, este sector ha presentado una aportación relevante a la demanda y a la oferta española. La intensa crisis económica que ha soportado la economía española en los últimos años ha influido 
indudablemente en el sector hostelería y turismo, especialmente en el consumo interno, aunque las peculiaridades de muchas de las actividades de este sector han hecho posible un mejor comportamiento cíclico en las actividades turísticas. En este sentido, resulta necesario resaltar que la actividad turística está contribuyendo de forma muy positiva a la recuperación económica de la economía española tras la crisis padecida. Por tanto, el turismo se ha convertido en un sector fundamental en la economía española.

Lo anterior puede haber incentivado la actividad investigadora sobre el sector Hostelería y Turismo en España. De hecho, habiendo acotado el estudio a las tesis doctorales registradas con el código UNESCO 531213 Sector Hostelería y Turismo, por ser trabajos inéditos y extensos sobre el campo de conocimiento objeto de estudio, se ha constatado como dichas investigación han proliferado en los últimos años, experimentando un intenso crecimiento exponencial, habiendo alcanzado cifras muy destacadas en el último período, lo que viene acompañado no sólo por el interés que suscita este sector en la economía española, por su enorme dimensión y dinamismo, sino también por la inclusión de actividades formativas sobre el sector turístico en el ámbito universitario. En efecto, la incorporación de los estudios de Turismo como titulación universitaria, y su posterior adscripción a los estudios de posgrado y doctorado, creó la necesidad de profesorado altamente especializado en esta línea de investigación, con competencias no sólo formativas sino también investigadoras, y con la titulación de doctor, que le permitiera impartir con una cierta dosis de éxito la docencia universitaria en los centros universitarios españoles. En efecto, teniendo en cuenta lo expuesto en el párrafo precedente, en general, cabe afirmar que las tesis doctorales han supuesto una vía interesante de investigación del sector del turismo reflejado por la creciente producción científica a lo largo del tiempo, tal y como constatan los estudios previamente citados en la revisión de la literatura realizada en este trabajo.

Con respecto al género de los investigadores, si bien es cierto que las investigaciones en el sector objeto de estudio de las mujeres es más tardía, como se ha podido comprobar, la producción científica en los últimos años ha sido, sin duda, mucho más prolífica, provocando que, durante el periodo analizado, y sobre todo en las últimas dos décadas, el número de tesis doctorales defendidas por hombres y por mujeres hayan sido prácticamente iguales (264 tesis defendidas por hombres y 263 tesis defendidas por mujeres).

Atendiendo a la producción científica de las universidades en este campo científico, existen algunas que han destacado por el número de tesis elaboradas y leídas en su seno, lo que origina una cierta concentración de la producción científica en pocas universidades españolas. Así, la Universidad de Málaga es la que registra un mayor número de tesis doctorales en la base de datos TESEO (62 tesis doctorales registradas). Le siguen a cierta distancia la Universidad de las Illes Balears (con 48), la Universidad de Alicante (con 27), la Universidad de La Laguna (con 26), la Universidad de Girona (con 25), y la Universidad de Sevilla (con 20), lo que supone casi un $40 \%$ de las tesis defendidas en las universidades españolas en el periodo 1978-2018. Cabe resaltar que se trata de universidades en las que el impacto de esta disciplina tiene una especial relevancia por su ubicación, ya que se trata de zonas en las que económica y culturalmente las actividades turísticas tienen un determinado peso específico, tal y como concluyen Vargas (2011b) y Moreno y Picazo (2012). 
El idioma generalmente empleado por la inmensa mayoría de los autores de tesis defendidas en el periodo analizado ha sido el castellano. De hecho, de las 527 tesis registradas 474 se presentaron en español, lo que supone cerca de un $90 \%$ de las tesis totales registradas. No obstante, resulta necesario añadir que cada vez se presentan más tesis en un idioma distinto al castellano, como se ha podido detectar en el estudio realizado. Y, de todas las tesis defendidas en el periodo en cuestión, 44 han obtenido la Mención Europea o Mención Internacional, de las que 24 fueron defendidas por hombres y 20 por mujeres.

Con respecto a la productividad de los directores de tesis en el Sector Hostelería y Turismo, el Profesor Torres Bernier encabeza el listado de profesores con mayor volumen de direcciones de tesis, con 13 tesis doctorales dirigidas en la Universidad de Málaga. Al Profesor Torres Bernier le sigue el Profesor Vera Rebollo, con 9 tesis doctorales dirigidas, y el Profesor Guía Julve, con 8 tesis doctorales. No obstante, resulta conveniente mostrar la atención en la evolución experimentada por la codirección de tesis doctorales, que a partir del año 2008 comienza a experimentar una evolución creciente en este campo científico.

El Profesor Aguiló Pérez fue el que más veces viene registrado como presidente en los tribunales que han juzgado las tesis doctorales correspondientes a este campo científico, con 18 tribunales, en diferentes universidades. Le siguen el Profesor Vera Rebollo, con 9 tribunales de tesis, y la Profesora Parra Guerrero, con 7 tribunales.

Lo anterior demuestra la existencia un profesorado universitario muy implicado en este campo científico, que ha participado muy activamente en la evolución científica en este campo del saber, invirtiendo gran parte de su tiempo y esfuerzo investigador en la dirección de tesis doctorales y acompañamiento en la investigación del doctorado, la lectura minuciosa previa de los documentos presentados por el doctorando en la fase de elaboración de las tesis doctorales y posterior evaluación y valoración de estos trabajos de investigación en este campo científico.

Respecto al género de los/as directores/as de tesis, se constata como la mayoría de las tesis registradas en la base de datos objeto de estudio han sido dirigidas por hombres. No obstante, en los últimos años, el número de directoras de tesis se ha incrementado sustancialmente, lo que viene reduciendo las diferencias existentes en este aspecto analizado. Del mismo modo, la evolución ha sido similar en el caso de los/as codirectores/as de tesis, como de los tribunales de tesis que han juzgado los trabajos de investigación defendidos en este campo.

Este documento ha seguido la senda de un riguroso análisis bibliométrico científico. No obstante, no está exento de algunas limitaciones. Una de ellas es la muestra de tesis doctorales seleccionadas, las de la base de datos TESEO. Existen otras bases de datos y algunas tesis no se registran en dicha base de datos. No obstante, por ser la inmensa mayoría las que se registran en dicha base de datos, y contener los datos que se trataba de analizar, se decidió finalmente por explotar dicha información. Otra de las limitaciones era la de seleccionar el descriptor, pues no todas las tesis de turismo se habrán catalogado en dicho descriptor, teniendo en cuenta que el turismo es un área de conocimiento multidisciplinar (Law et al., 2009; Huang, 2011; Chang y McAleer, 2012). No obstante, resultaba necesario acotar el descriptor de análisis para la selección de la muestra a estudiar. 
Finalmente, es necesario poner de manifiesto que a pesar de las conclusiones vertidas por este trabajo de investigación, resulta conveniente añadir que el análisis llevado a cabo no concluye en estos momentos, sino que sigue vigente, con la finalidad de profundizar en esta línea de trabajo y de abrir nuevas vías de análisis que puedan ser abordadas. Consecuentemente, es fundamental resaltar la necesidad de seguir avanzando en el estudio de algunas cuestiones tratadas en este documento y profundizar en otros aspectos que no han sido abordados aún, a partir de la explotación más intensa de la base de datos usada, así como de otras fuentes de información, que permitan ahondar en las reflexiones realizadas y obtener nuevos resultados no tratados de forma pormenorizada en esta investigación. $Y$ los investigadores, así como los centros e instituciones de investigación, deben seguir avanzando en la discusión de estas cuestiones que benefician, sin duda, al progreso del conocimiento.

\section{REFERENCIAS BIBLIOGRÁFICAS}

Agencia Valenciana del Turismo (2006): Análisis de las tesis doctorales de turismo realizadas en universidades españolas. Papers de Turisme, 40, 111-116.

Albacete, C.A., y Fuentes, M.M. (2010): Difusión de la investigación española sobre turismo en revistas internacionales. Análisis Turístico, 9, 14-29.

Albacete, C.A.; Fuentes, M.M.; Haro-Domínguez, M.C. (2010): La investigación española en turismo con impacto internacional (1997-2011). Una perspectiva desde la economía y la dirección de empresa". Cuadernos de Economía y Dirección de la Empresa, 16 (1), 17-28.

Araújo, N., y Fraiz, J.A. (2013): La formación turística en España: evolución y oferta universitaria actual. Cuaderno Virtual de Turismo, 13(3), 289-307.

Bermúdez, M.P., y Castro, A.; Sierra, J.C.; Buela-Casal, G. (2009): Análisis descriptivo transnacional de los estudios de doctorado en el EEES. Revista de Psicodidáctica, 14 (2), 193-210.

Bravo, S. (2004): La competitividad del sector turístico. Boletín Económico del Banco de España, 9/2004, 89-106.

Ceballos, C., Arias, C., Ruiz, A., Sanz, C., y Vázquez, I. (2010): La formación en turismo en España: pasado, presente y futuro en el nuevo Espacio Europeo de Educación Superior. Cuadernos de Turismo, 25, 45-67.

Chang, C.; McAleer, M. (2012): Citations and impact of ISI tourism and hospitality journals. Tourism Management Perspectives, 1, 2-8.

Colás, M.P., Buendía, L., y Hernández, F. (2009): Competencias científicas para la realización de una tesis doctoral. Barcelona: Editorial Davinci.

Conferencia de Rectores de Universidades Españolas (CRUE) (2013): La universidad española en cifras 2012. Madrid: Cátedra UNESCO de Gestión y Política Universitaria de la Universidad Politécnica de Madrid.

Corral-Marfil, J.A.; Cánoves-Valiente, G. (2016): An Approach to Tourism Research in Spain, en Munar, A.M. y Jamal, T. (ed.), Tourism Research Paradigms: Critical and Emergent Knoledges, Esmerald Group Publishing Limited, 167-189.

Cortés, D. (2007): Medir la producción científica de los investigadores universitarios: la bibliometría y sus límites. Revista de la Educación Superior, Vol. XXXVI (2), no 142, 43-65.

Das, A.; Handfield, R. (1997): A Meta-Analysis of Doctoral Dissertations in Purchasing. Journal of Operations Management, 15 (2), 101-121. 
Durán, A.; Álvarez, J.; Cruz del Río, M. (2015): Active tourism research: a literatura review. ROTUR, Revista de Ocio y Turismo, 8, 62-76.

Esteban, A. (2000): La investigación turística en la Universidad Española. Estudios Turísticos, $144-145,155-180$.

Esteban, A. (2004): Modelos de la demanda turística en España: segmentación por país de procedencia. Mediterráneo Económico, 5, 81-101.

Fernández-Cano, A., Torralbo, M., y Vallejo, M. (2008): Revisión y prospectiva de la producción española en tesis doctorales de Pedagogía (1976-2006). Revista de Investigación Educativa, 26(1), 191-208.

Flores, D., y Barroso, M.O. (2012): La demanda turística internacional. Medio siglo de evolución. Revista de Economía Mundial, 32, 127-149.

Fuentes, E., y Arguimbau, L. (2010): Las tesis doctorales en España (1997-2008): análisis, estadísticas y repositorios cooperativos. Revista Española de Documentación Científica, 33(1), 63-89 (https://doi.org/10.3989/redc.2010.1.711).

Hall, C.M. (2011): Publish and perish? Bibliometric analysis, journal ranking and the assessment of research quality in tourism. Tourism Management, 32 (1), 16-27.

Helmsing, A.H.J., y Ellinger, P. (2011). La economía política institucional del desarrollo local: dos cuentos de turismo en Brasil. Eure, 37(110), 31-57 (https://doi.org/10.4067/ S0250-71612011000100002).

Hernández, J.M.; Campón, A.M.; Folgado, J.A. (2011): La investigación en Turismo en España a través de las revistas de Dirección de Empresas. Análisis Turístico, 11, 1-9.

Hostelsur (2018): Recuperado de https://www.hosteltur.com/111389_turismo-genera-98-economia-mundial.html.

Jeong, W-S. (2001): Location Information Science: Change in Ph. D. Dissertations during the Past Three Decades. Journal of Education for Library and Information Sciences, 42 (4), 308-324.

Jiménez, M., Peña, A.R., y Ruiz, J. (2015a): Factores incidentes en la recuperación turística en la actual crisis en las diferentes regiones europeas, un análisis estático. Revista de Estudios Regionales, 104, 33-57.

Jiménez, M., Ruiz, J., y Peña, A.R. (2015b): Análisis de las zonas rurales andaluzas y su turismo, desde una óptica de proximidad geográfica a los núcleos urbanos andaluces. Investigaciones Regionales, 31, 59-74.

Jiménez, M., Peña, A.R., y Ruiz, J. (2016): Bipolaridad turística en Europa. La consolidación de destinos maduros en épocas de crisis. Cuadernos de Turismo, 38, 221-243 (https://doi. org/10.6018/turismo.38.271431).

Jiménez, M., Peña, A.R., y Ruiz, J. (2017): La demanda turística internacional: recuperación de la crisis y turismo de lujo, una primera aproximación al caso español. Investigaciones Regionales, 38, 47-66.

Law, R.; Ye, Q.; Chen, W.; Leung, R. (2009): An Analysis of the most Influential Articles Published in Tourism Journals from 2000 to 2007: A Google Scholar Approach. Journal of Travel and Tourism Marketing, 26 (7), 735-746.

Lillo, A., Ramón, A.B., y Sevilla, M. (2009): El capital humano como factor estratégico para la competitividad del sector turístico. Cuaderno de Turismo, 19, 47-69. 
López, J.M.; López, L.M. (2008): Producción científica española en marketing turístico. Estudios Turísticos, 177, 35-50.

López-Bonilla, J.M.; Granados-Perea, C.; López-Bonilla, L.M. (2017): Primera generación de autores con difusión internacional en la investigación turística española. Revista española de Documentación Científica, 40 (3), 1-18 (https://dx.doi.org/10.3989/ redc.2017.3.1399).

Martínez, M.J. (2011a): Evaluación de la producción científica sobre publicidad (1971-2001) (Tesis Doctoral). Universidad Complutense de Madrid.

Martínez, M.J. (2011b): La investigación universitaria en Publicidad: producción y temática de las tesis doctorales (1971-2001). Documentación de las Ciencias de la Información, 34, 119-156 (https://doi.org/10.5209/rev DCIN.2011.v34.36449).

Massieu, A. (2004): El sector turístico visto desde la OMT: una reflexión a partir de la coyuntura reciente a nivel mundial. En Uriel, E. y Hernández, R. (Eds.), Análisis y tendencias del turismo (pp, 19-44). Madrid: Pirámide.

Miguel, A. (2000): Aportaciones al estudio de la literatura gris universitaria: La evolución de las tesis doctorales en España. Primer Congreso Universitario de Ciencias de la Documentación: Teoría, historia y metodología de las ciencias de la documentación (pp. 645-651). Madrid: Universidad Complutense de Madrid.

Moreno-Fernández, O., y Moreno-Crespo, P. (2016): Análisis bibliométrico de las tesis doctorales españolas indexadas con el descriptor "Sector de la educación" (1976/2014). Revista Española de Documentación Cientifica, 39(3), 1-14.

Moreno, S., y Picazo, P. (2012): Difusión de la investigación científica en revistas de turismo realizada por instituciones españolas. Revista de Análisis Turístico, 14(2), 33-52.

Muñoz, A. (2005): The Scholarly Transition of Female Academics at the University of Granada (1975-1990). Scientometrics, 64 (3), 325-350.

Navarro, P., y Vejsberg, L. (2009). El proyecto turístico barilochense antes de Bustillo: entre la prehistoria del Parque Nacional Nahuel Huapi y el desarrollo local. Estudios y perspectivas en Turismo, 18(4), 414-433.

Nel-lo, M., y Pérez, Y. (2007): La política turística en Panamá. Resultados y perspectivas. Cuadernos de Turismo, 20, 199-221.

Organización Mundial del Turismo (OMT) (2017): Panorama OMT del turismo internacional. Madrid, Edición 2017. Recuperado de https://www.eunwto.org/doi/ pdf/10.18111/978928-4419043.

Ortega, E.; Rodríguez, B.; Cobo, F. (2003): La investigación del turismo a través de las tesis doctorales. Un análisis comparativo entre España, Estados Unidos y Francia, en Ortega, E. (Coord.), Investigación y Estrategias turísticas. Thomson, Madrid, 65-89.

Ortega, E., y Rodríguez, B. (2004): La investigación turística a través de tesis doctorales. Un análisis comparativo entre España y Francia. Estudios Turísticos, no 159, 5-27.

Ortiz-Sánchez, S., y Martín-Moreno, C. (2011): Las tesis doctorales como instrumento para conocer la evolución de la producción en biblioteconomía y documentación: el caso del Departamento de Biblioteconomía y Documentación de la Universidad Carlos III. Investigación Bibliotecológica, 25 (55), 151-174.

Precedo, A., Revilla, A., y Míguez, A. (2007). El turismo cultural como factor estratégico de desarrollo: el camino de Santiago. Estudios Geográficos, LXVIII (262), 205-234. 
Racherla, P., y Hu, C. (2010): A social network perspective of tourism research collaborations. Annals of Tourism Research, 37 (4), 1012-1034.

Repiso, R., Torres, D., y Delgado, E. (2011): Análisis de la investigación sobre Radio en Espa-a: una aproximación a través del Análisis Bibliométrico y de Redes Sociales de las tesis doctorales defendidas en España entre 1976-2008. Estudios sobre el mensaje periodístico, 17(2), 417-429.

Rosselló, J., Aguiló, E., y Riera, A. (2005): Un modelo dinámico de demanda turística para Baleares. Revista de Economía Aplicada, 39, 5-20.

Salvador, J. (2007): Top ten. Diez años de investigación española en Biblioteconomía y Documentación (1996-2006). Ranking e índice $\mathrm{h}$ de los diez autores en activo más citados. Revista General de Información y Documentación, 17 (2), 159-182.

Soli, A., y Merlo, J.A. (2002): Bases de datos y recursos en Internet de tesis doctorales. Revista Española de Documentación Científica, 25(1), 95-106.

Huang, S. (2011): Tourism as the Subject of China's Doctoral Dissetations. Annals of Tourism Research, 38, 309-330 (https://doi:10.1016/i.annals.2010.08.005).

TESEO (Ministerio de Educación, Cultura y Deporte). Recuperado de https://www.educacion. gob.es/teseo/irGestionarConsulta.do (Consultada hasta el mes de marzo de 2018).

Torres, E. (1994): Turismo y desarrollo regional. Papers de Turisme, 14-15, 95-102.

Vargas, A. (2011a): Tourism research in Spain: the state of the art. Enlightening Tourism, $A$ Pathmaking Journal, 1, 93-110.

Vargas, A. (2011b): ¿Los principales destinos son también las principales potencias en la investigación en turismo? Estudios Turísticos, 188, 91-111.

Vera, J.F., e Ivars, J.A. (2001): La formación y la investigación turística en España: una visión de síntesis. Papers de Turisme, 29, 6-27.

Xiao, H.; Smith, S.J.L. (2008): Knowledge Impact an Appraisal of Tourism Scholarship. Annals of Tourism Research, 35 (1), 62-83.

Zhao, W.; Richie, J.R.B. (2007): An Investigation of Academic Leadership in Tourism Research: 1985-2004. Tourism Management, 28 (2), 476-490. 


\section{Anexo I}

Número de tesis por universidades, sexo, idiomas, y mención

\begin{tabular}{|c|c|c|c|c|c|c|}
\hline \multirow{2}{*}{ UNIVERSIDAD } & \multirow{2}{*}{ № Tesis } & \multirow{2}{*}{ \% Tesis } & \multicolumn{2}{|c|}{$\begin{array}{l}\text { Número de tesis según } \\
\text { género }\end{array}$} & \multicolumn{2}{|c|}{$\begin{array}{l}\text { Idioma y mención } \\
\text { europea/mención } \\
\text { internacional }\end{array}$} \\
\hline & & & Mujeres & Hombres & $\begin{array}{c}\text { № tesis en } \\
\text { idiomas }\end{array}$ & $\begin{array}{c}\text { № de } \\
\text { menciones }\end{array}$ \\
\hline Universidad de Málaga & 62 & 11,76 & 32 & 30 & 3 & 6 \\
\hline Universidad de las Illes Balears & 48 & 9,11 & 19 & 29 & 10 & 2 \\
\hline Universidad de Alicante & 27 & 5,12 & 17 & 10 & 0 & 0 \\
\hline Universidad de La Laguna & 26 & 4,93 & 10 & 16 & 1 & 3 \\
\hline Universidad de Girona & 25 & 4,74 & 13 & 12 & 10 & 1 \\
\hline Universidad de Sevilla & 20 & 3,8 & 11 & 9 & 0 & 1 \\
\hline Univ. Politécnica de Valencia & 19 & 3,61 & 7 & 12 & 1 & 2 \\
\hline Universidad de Extremadura & 18 & 3,42 & 7 & 11 & 1 & 1 \\
\hline Universidad Antonio de Nebrija & 16 & 3,04 & 9 & 7 & 2 & 0 \\
\hline Univ. Santiago de Compostela & 16 & 3,04 & 10 & 6 & 5 & 4 \\
\hline Universidad de Vigo & 14 & 2,66 & 8 & 6 & 1 & 1 \\
\hline Universidad de Granada & 13 & 2,47 & 6 & 7 & 0 & 4 \\
\hline Univ. Complutense de Madrid & 12 & 2,28 & 6 & 6 & 0 & 2 \\
\hline Universidad de Barcelona & 12 & 2,28 & 5 & 7 & 0 & 0 \\
\hline Universidad de Valencia & 12 & 2,28 & 6 & 6 & 0 & 2 \\
\hline Universidad de A Coruña & 11 & 2,09 & 7 & 4 & 0 & 2 \\
\hline Univ. Las Palmas de G. Canaria & 11 & 2,09 & 5 & 6 & 0 & 0 \\
\hline Universidad de Salamanca & 11 & 2,09 & 5 & 6 & 4 & 0 \\
\hline Universidad de Deusto & 10 & 1,9 & 8 & 2 & 1 & 2 \\
\hline Universidad Rovira I Virgili & 10 & 1,9 & 7 & 3 & 2 & 0 \\
\hline Univ. Autónoma de Barcelona & 9 & 1,71 & 3 & 6 & 1 & 3 \\
\hline Universidad de Zaragoza & 9 & 1,71 & 5 & 4 & 0 & 2 \\
\hline Univ. Autónoma de Madrid & 7 & 1,33 & 3 & 4 & 1 & 1 \\
\hline Univ. de Castilla-La Mancha & 6 & 1,14 & 5 & 1 & 0 & 0 \\
\hline Universidad de Valladolid & 6 & 1,14 & 5 & 1 & 1 & 0 \\
\hline Univ. Jaume I de Castellón & 6 & 1,14 & 3 & 3 & 0 & 0 \\
\hline Universidad Rey Juan Carlos & 6 & 1,14 & 4 & 2 & 0 & 0 \\
\hline Universidad de Cádiz & 5 & 0,95 & 1 & 4 & 0 & 1 \\
\hline Universidad de Cantabria & 5 & 0,95 & 3 & 2 & 0 & 1 \\
\hline Universidad de Jaén & 5 & 0,95 & 2 & 3 & 0 & 1 \\
\hline Univ. Miguel Hernández Elche & 5 & 0,95 & 2 & 3 & 0 & 0 \\
\hline Universitat Abat Oliva CEU & 5 & 0,95 & 2 & 3 & 1 & 0 \\
\hline Univ. Católica San Antonio & 4 & 0,76 & 2 & 2 & 0 & 0 \\
\hline Universidad de Oviedo & 4 & 0,76 & 3 & 1 & 0 & 0 \\
\hline Univ. Politécnica de Madrid & 4 & 0,76 & 0 & 4 & 0 & 0 \\
\hline
\end{tabular}


La producción científica en el "Sector de Hostelería y Turismo": Un análisis bibliométrico de las tesis doctorales españolas en el periodo 1978-2018

\begin{tabular}{|c|c|c|c|c|c|c|}
\hline Universidad Camilo José Cela & 3 & 0,57 & 2 & 1 & 0 & 0 \\
\hline Univ. Carlos III de Madrid & 3 & 0,57 & 2 & 1 & 1 & 0 \\
\hline Universidad de Almería & 3 & 0,57 & 1 & 2 & 0 & 0 \\
\hline Universidad de León & 3 & 0,57 & 1 & 2 & 0 & 1 \\
\hline Universidad del País Vasco & 3 & 0,57 & 2 & 1 & 0 & 0 \\
\hline Universidad Pablo de Olavide & 3 & 0,57 & 1 & 2 & 0 & 0 \\
\hline Universidad Pompeu Fabra & 3 & 0,57 & 2 & 1 & 1 & 0 \\
\hline Univ. Valencia (Estudi General) & 3 & 0,57 & 1 & 2 & 0 & 0 \\
\hline Universidad de Alcalá & 2 & 0,38 & 1 & 1 & 0 & 0 \\
\hline Universidad de Córdoba & 2 & 0,38 & 1 & 1 & 0 & 1 \\
\hline Universidad de Lleida & 2 & 0,38 & 1 & 1 & 0 & 0 \\
\hline Univ. Intern. de Andalucía & 2 & 0,38 & 1 & 1 & 0 & 0 \\
\hline Univ. N. E. a Distancia (UNED) & 2 & 0,38 & 1 & 1 & 0 & 0 \\
\hline Univ. Santiago de Compostela & 2 & 0,38 & 1 & 1 & 0 & 0 \\
\hline IE Universidad & 1 & 0,19 & 1 & 0 & 0 & 0 \\
\hline Universidad Cardenal Herrera & 1 & 0,19 & 0 & 1 & 0 & 0 \\
\hline U. Católica Sta. Teresa de Jesús & 1 & 0,19 & 1 & 0 & 0 & 0 \\
\hline Universidad de Huelva & 1 & 0,19 & 0 & 1 & 0 & 0 \\
\hline Universidad de Murcia & 1 & 0,19 & 1 & 0 & 0 & 0 \\
\hline Universidad de San Pablo & 1 & 0,19 & 0 & 1 & 0 & 0 \\
\hline Univ. Politécnica de Cartagena & 1 & 0,19 & 0 & 1 & 0 & 0 \\
\hline Univ. Politécnica de Cataluña & 1 & 0,19 & 0 & 1 & 0 & 0 \\
\hline Univ. Pontificia de Salamanca & 1 & 0,19 & 0 & 1 & 0 & 0 \\
\hline Universidad Ramón Llull & 1 & 0,19 & 0 & 1 & 0 & 0 \\
\hline Universidad San Pablo-CEU & 1 & 0,19 & 1 & 0 & 0 & 0 \\
\hline Univ. Oberta de Catalunya & 1 & 0,19 & 0 & 1 & 1 & 0 \\
\hline TOTAL & 527 & 100,00 & 263 & 264 & 48 & 44 \\
\hline
\end{tabular}

FUENTE: Elaboración propia a partir de los datos de la Base de datos TESEO. 


\section{Anexo II}

\section{Directores de tesis más productivos en Sector de hostelería y Turismo}

\begin{tabular}{|c|c|c|c|}
\hline Director/a & № Tesis & Universidad & $\begin{array}{c}\text { Mención } \\
\text { Europea/ } \\
\text { Internacional }\end{array}$ \\
\hline Torres Bernier, Enrique & 13 & Universidad de Málaga & \\
\hline Vera Rebollo, José Fernando & 9 & Universidad de Alicante & \\
\hline Guía Julve, Jaime Óscar & 8 & $\begin{array}{l}\text { Universidad de Girona (7) } \\
\text { Universitat Oberta de Catalunya (1) }\end{array}$ & \\
\hline Rey-Maquieira Palmer, Javier & 7 & Universidad de Las Illes Balears & \\
\hline Fraiz Brea, José Antonio & 6 & Universidad de Vigo & \\
\hline Díaz Pérez, Flora María & 5 & Universidad de La Laguna & 1 \\
\hline Santana Talavera, Agustín & 5 & $\begin{array}{l}\text { Universidad de La Laguna (4) } \\
\text { Universidad Pablo de Olavide (1) }\end{array}$ & 1 \\
\hline Serra Cantallops, Antoni & 5 & Universidad de Las Illes Balears & \\
\hline Martínez Fernández, Valentín A. & 4 & Universidad de A Coruña & \\
\hline Pulido Fernández, Juan Ignacio & 4 & Universidad de Jaén & 1 \\
\hline Tous Zamora, Dolores & 4 & Universidad de Málaga & \\
\hline Aguiló Pérez, Eugeni & 3 & Universidad de Las Illes Balears & \\
\hline Alegre Martín, Joaquín & 3 & Universidad de Las Illes Balears & \\
\hline Alen González, María Elisa & 3 & Universidad de Vigo & 1 \\
\hline Antón Clave, Salvador & 3 & Universidad Rovira i Virgili & \\
\hline Cánoves Valiente, Gema & 3 & Univ. Autónoma de Barcelona & 1 \\
\hline Fernández Alles, María Teresa & 3 & Universidad de Cádiz & 1 \\
\hline Martín Jiménez, Isabel & 3 & Universidad de Salamanca & \\
\hline Mazón Martínez, Tomás & 3 & Universidad de Alicante & \\
\hline Nogués Pedregal, Antonio Miguel & 3 & Univ. Miguel Hernández de Elche & \\
\hline Vidal Casellas, María Dolors & 3 & Universidad de Girona & \\
\hline Alarcón Urbistondo, Pilar & 2 & Universidad de Málaga & 1 \\
\hline Alcaide Inchausti, Ángel & 2 & Univ. Complutense de Madrid & 1 \\
\hline Almeida García, Fernando Nelson & 2 & Universidad de Málaga & \\
\hline Álvarez Sousa, Antón & 2 & Universidad de A Coruña & 1 \\
\hline Aragón Cánovas, Francisco Javier & 2 & Universidad Camilo José Cela & \\
\hline Barroso González, María de la O & 2 & $\begin{array}{l}\text { Universidad de Huelva (1) } \\
\text { Univ. Internacional de Andalucía (1) }\end{array}$ & \\
\hline Bigne Alcañíz, José Enrique & 2 & Universidad de Valencia & \\
\hline Boned Purkiss, Javier & 2 & $\begin{array}{l}\text { Univ. Politécnica de Madrid (1) } \\
\text { Universidad de Málaga (1) }\end{array}$ & \\
\hline Bueno Campos, Eduardo & 2 & Universidad Autónoma de Madrid & \\
\hline Cabrero Diéguez, Valentín & 2 & Universidad de Salamanca & \\
\hline Canos Daros, Lourdes & 2 & Universitat Politécnica de Valencia & \\
\hline Cortés Puya, Trinidad & 2 & Universidad Antonio de Nebrija & \\
\hline Crespi Cladero, Rafel & 2 & Universidad de las Illes Balears & \\
\hline
\end{tabular}


La producción científica en el "Sector de Hostelería y Turismo": Un análisis bibliométrico de las tesis doctorales españolas en el periodo 1978-2018

\begin{tabular}{|c|c|c|c|}
\hline Díaz Méndez, Montserrat & 2 & Universidad de Extramadura & \\
\hline Esteban Tayala, Águeda & 2 & Universidad de Castilla-La Mancha & \\
\hline Fernández Cavia, Josep & 2 & Universidad Pompeu Fabra & \\
\hline Frías Jamilena, Dolores & 2 & Universidad de Granada & \\
\hline García Falcón, Juan Manuel & 2 & Univ. Las Palmas de Gran Canarias & \\
\hline García Pérez de Lema, Domingo & 2 & $\begin{array}{l}\text { Universidad de Cantabria (1) } \\
\text { Univ. Politécnica de Cartagena (1) }\end{array}$ & \\
\hline Guevara Plaza, Antonio & 2 & Universidad de Málaga & \\
\hline Gutiérrez Calderón, María Isabel & 2 & Univesidad Carlos III de Madrid & \\
\hline Hernández Mogollón, José Manuel & 2 & Universidad de Extremadura & 1 \\
\hline Hidalgo Moratal, Moisés & 2 & Universidad de Alicante & \\
\hline Ivars Baidal, Josep Antoni & 2 & Universidad de Alicante & \\
\hline Jafari, Jafar & 2 & Universidad de las Illes Balears & \\
\hline Latiesa Rodríguez, Bienvenida M. & 2 & Universidad de Granada & 1 \\
\hline Lois González, Rubén Camilo & 2 & Univ. de Santiago de Compostela & \\
\hline López Bonilla, Jesús Manuel & 2 & Universidad de Sevilla & \\
\hline Marchante Mera, Andrés & 2 & Universidad de Málaga & \\
\hline Martínez Roget, Fidel & 2 & Univ. de Santiago de Compostela & \\
\hline Matías Reche, Fernando & 2 & Universidad de Granada & 1 \\
\hline Miranda González, Francisco Javier & 2 & Universidad de Extremadura & \\
\hline Morere Molinero, Nuria & 2 & Universidad Rey Juan Carlos & \\
\hline Muñoz Mazón, Ana & 2 & $\begin{array}{l}\text { Universidad Antonio de Nebrija (1) } \\
\text { Universidad Rey Juan Carlos (1) }\end{array}$ & \\
\hline Navarro Jurado, Enrique & 2 & Universidad de Málaga & \\
\hline Oreja Rodríguez, Juan Ramón & 2 & Universidad de La Laguna & \\
\hline Parra Guerrero, Francisca & 2 & Universidad de Málaga & \\
\hline Peiró Silla, José María & 2 & $\begin{array}{l}\text { Universidad de Valencia (1) } \\
\text { Universidad Jaume I de Castellón }\end{array}$ & \\
\hline Pie Ninot, Ricard & 2 & Universidad de Málaga & \\
\hline Rastrollo Horrillo, Mạ Ángeles & 2 & Universidad de Málaga & \\
\hline Ripoll Alcón, Joan & 2 & Universitat Abat Oliba CEU & \\
\hline Rocafort Nicolau, Alfredo & 2 & $\begin{array}{l}\text { Universidad de Barcelona (1) } \\
\text { Universidad Rovira i Virgili (1) }\end{array}$ & \\
\hline Ruiz de la Rúa, Francisco & 2 & Universidad de Málaga & \\
\hline Ruiz Jiménez, Antonio & 2 & Universidad de Sevilla & \\
\hline Santana Turegano, Manuel Ángel & 2 & Universidad de La Laguna & \\
\hline Santos Solla, Xose Manuel & 2 & Univ. de Santiago de Compostela & 1 \\
\hline Seguí Llinas, Miquel & 2 & Universidad de las Illes Balears & \\
\hline Serra Busquets, Sebastia & 2 & Universidad de las Illes Balears & \\
\hline Simancas Cruz, Moisés R. & 2 & Universidad de La Laguna & \\
\hline Troitiño Minuesa, Miguel Ángel & 2 & Univ. Complutense de Madrid & \\
\hline
\end{tabular}

FUENTE: Elaboración propia a partir de los datos de la Base de datos TESEO. 


\section{Anexo III}

\section{Presidentes de Tribunal con mayor número de tesis}

\begin{tabular}{|c|c|c|}
\hline Presidente de Tribunal & № Tesis & Universidades (№ Tesis) \\
\hline Aguiló Pérez, Eugeni & 18 & $\begin{array}{l}\text { Universidad de Jaén (3) } \\
\text { Universidad de las Illes Balears (11) } \\
\text { Universidad de Málaga (2) } \\
\text { Universidad de Oviedo (1) } \\
\text { Universidad Jaume I de Castellón (1) }\end{array}$ \\
\hline Vera Rebollo, José Fernando & 9 & $\begin{array}{l}\text { Universidad Antonio de Nebrija (1) } \\
\text { Universidad de Alicante (1) } \\
\text { Universidad de Girona (1) } \\
\text { Universidad de Málaga (2) } \\
\text { Universidad de Oviedo (1) } \\
\text { Universidad de Sevilla (1) } \\
\text { Universidad de Vigo (1) } \\
\text { Universitat Politécnica de Valencia (1) }\end{array}$ \\
\hline Parra Guerrero, Francisca & 7 & Universidad de Málaga (7) \\
\hline Valls Passola, Jaume & 6 & Universidad de Girona (6) \\
\hline Vázquez Casielles, Rodolfo & 6 & $\begin{array}{l}\text { Universidad de Alicante (1) } \\
\text { Universidad de Cantabria (2) } \\
\text { Universidad de Castilla-La Mancha (1) } \\
\text { Universidad de León (1) } \\
\text { Universidad de Oviedo (1) }\end{array}$ \\
\hline Campesino Fernández, Antonio-José & 5 & $\begin{array}{l}\text { Universidad de Extremadura (1) } \\
\text { Universidad de Málaga (3) } \\
\text { Universidad de Salamanca (1) }\end{array}$ \\
\hline Galán González, José Luis & 5 & $\begin{array}{l}\text { Universidad Carlos III de Madrid (1) } \\
\text { Universidad de Málaga (2) } \\
\text { Universidad de Sevilla (2) }\end{array}$ \\
\hline Nicolau Gonzálbez, Juan Luis & 5 & $\begin{array}{l}\text { Universidad de Alicante (1) } \\
\text { Universidad de Deusto (1) } \\
\text { Universidad de Girona (1) } \\
\text { Universidad Pompeu Fabra (1) } \\
\text { Universidad Rovira i Virgili (1) }\end{array}$ \\
\hline Pulido Fernández, Juan Ignacio & 5 & $\begin{array}{l}\text { Universidad Antonio de Nebrija (4) } \\
\text { Universidad de Málaga (1) }\end{array}$ \\
\hline Aguirre Sádaba, Aquilino Alfredo & 4 & Universidad de Málaga (4) \\
\hline Azqueta Oyarzun, Diego & 4 & $\begin{array}{l}\text { Universidad de Las Illes Balears (2) } \\
\text { Universidad de Las Palmas de Gran Canaria (1) } \\
\text { Universidad de Zaragoza (1) }\end{array}$ \\
\hline Fraiz Brea, José Antonio & 4 & $\begin{array}{l}\text { Universidad de Málaga (2) } \\
\text { Universidad de Vigo (2) }\end{array}$ \\
\hline Guevara Plaza, Antonio & 4 & $\begin{array}{l}\text { Universidad de Deusto (1) } \\
\text { Universidad de Málaga (1) } \\
\text { Universidad de Sevilla (1) } \\
\text { Universidad Jaume I de Castellón (1) }\end{array}$ \\
\hline López Palomeque, Francisco & 4 & $\begin{array}{l}\text { Universidad Autónoma de Barcelona (2) } \\
\text { Universidad de Alicante (2) }\end{array}$ \\
\hline Luque Martínez, Teodoro & 4 & $\begin{array}{l}\text { Universidad de Granada (2) } \\
\text { Universidad de Granada (1) } \\
\text { Universidad de Las Palma de Gran Canaria (1) }\end{array}$ \\
\hline
\end{tabular}


La producción científica en el "Sector de Hostelería y Turismo": Un análisis bibliométrico de las tesis doctorales españolas en el periodo $1978-2018$

\begin{tabular}{|c|c|c|}
\hline Ortigueira Bouzada, Manuel & 4 & $\begin{array}{l}\text { Universidad de Jaén (1) } \\
\text { Universidad de Málaga (3) }\end{array}$ \\
\hline Rodríguez Antón, José Miguel & 4 & $\begin{array}{l}\text { Universidad Autónoma de Madrid (1) } \\
\text { Universidad de A Coruña (1) } \\
\text { Universidad de Extremadura (1) } \\
\text { Universidad de Murcia (1) }\end{array}$ \\
\hline Alcaide Inchausti, Ángel & 3 & Universidad Complutense de Madrid (3) \\
\hline Antón Clave, Salvador & 3 & $\begin{array}{l}\text { Universidad de Alicante (1) } \\
\text { Universidad de Barcelona (1) } \\
\text { Universidad de Deusto (1) }\end{array}$ \\
\hline Bigne Alcañíz, José Enrique & 3 & $\begin{array}{l}\text { Universidad de Deusto (1) } \\
\text { Universidad de La Laguna (1) } \\
\text { Universitat de Valencia (Estudi General) (1) }\end{array}$ \\
\hline Cabero Diéguez, Valentín & 3 & Universidad de Salamanca (3) \\
\hline Corona Ramón, Juan Francisco & 3 & Universitat Abat Oliba CEU (3) \\
\hline Espejo Marín, Cayetano & 3 & $\begin{array}{l}\text { Universidad de Alicante (1) } \\
\text { Universidad de La Laguna (1) } \\
\text { Universidad de Salamanca (1) }\end{array}$ \\
\hline Esteban Talaya, Águeda & 3 & $\begin{array}{l}\text { Universidad de Castilla-La Mancha (1) } \\
\text { Universidad de Extremadura (1) } \\
\text { Universidad de Salamanca (1) }\end{array}$ \\
\hline González Vázquez, Encarnación & 3 & $\begin{array}{l}\text { Universidad de Vigo (2) } \\
\text { Universidad Rey Juan Carlos (1) }\end{array}$ \\
\hline Latiesa Rodríguez, Bienvenida M. & 3 & Universidad de Málaga (3) \\
\hline Leal Millán, Antonio Genaro & 3 & $\begin{array}{l}\text { Universidad de Extremadura (1) } \\
\text { Universidad de Granada (2) }\end{array}$ \\
\hline Martín Armario, Enrique & 3 & $\begin{array}{l}\text { Universidad de Extremadura (2) } \\
\text { Universidad de Vigo (1) }\end{array}$ \\
\hline Pascual Fernández, José Jaime & 3 & $\begin{array}{l}\text { Universidad de La Laguna (2) } \\
\text { Universidad Pablo de Olavide (1) }\end{array}$ \\
\hline Polo Redondo, Yolanda & 3 & Universidad de Zaragoza (3) \\
\hline Troitiño Vinuesa, Miguel Ángel & 3 & $\begin{array}{l}\text { Universidad Complutense de Madrid (1) } \\
\text { Universidad de Alicante (1) } \\
\text { Universidad de Santiago de Compostela (1) }\end{array}$ \\
\hline Vargas Sánchez, Alfonso & 3 & $\begin{array}{l}\text { Universidad de Extremadura (1) } \\
\text { Universidad de Sevilla (1) } \\
\text { Universidad de Vigo (1) }\end{array}$ \\
\hline
\end{tabular}

FUENTE: Elaboración propia a partir de los datos de la Base de datos TESEO. 